\title{
AS CÔNICAS E SUAS CONSTRUÇÕES GEOMÉTRICAS
}

\author{
Sarley de Araújo Silva ${ }^{1}$ \\ Josimauro Borges de Carvalho ${ }^{2}$
}

RESUMO: A Geometría das cónicas está presente em diversas áreas do conhecimento, seja nas engenharías, nos elementos da natureza em objetos geométricos do cotidiano. $\mathrm{O}$ objetivo deste trabalho e confeccionar figuras geométricas com materiais concretos como recurso pedagógico a partir da definição, com o intuito de tornar mais significativa e presente as cônicas geométricas: parábola, elipse e hipérbole na sala de aula, valorizando os saberes anteriores dos educandos, proporcionando-lhes o raciocínio geométrico e matemático. $\mathrm{Na}$ realização desta temática foi utilizado o método dedutivo e a pesquisaação. Os resultados mostram que o uso de material concreto como recurso pedagógico facilita o processo de ensino e aprendizagem em geometria das cônicas promovendo o raciocínio e o desenvolvimento do aluno. Portanto, concluí-se que as atividades com materiais manipuláveis seguindo regras matemáticas contribui na construção do conhecimento.

Palavras-Chaves: Cônicas. Construções geométricas. Hipérbole e geometria analítica.

ABSTRACT: Conics geometry is present in several areas of knowledge, whether in engineering, in the elements of nature or geometric everyday objects. The objective of this work is to make geometric figures with concrete materials as a pedagogical resource from the definition, in order to make the geometric conics more significant and present: parabola, ellipse and hyperbole in the classroom, valuing the students' previous knowledge, providing geometric and mathematical reasoning to them. In carrying out this theme, the deductive method and action research were used. The results show that the use of concrete material as a pedagogical resource facilitates the teaching and learning process in conic geometry, promoting student reasoning and development. Therefore, it is concluded that activities with manipulable materials following mathematical rules contribute to the construction of knowledge.

Keyword's: Conics. Geometric constructions. hyperbole and analytic geometry.

\section{INTRODUÇÃO}

No estudo das cônicas em geometria analítica, os educandos possuem dificuldade de entender os conceitos e aplicações que envolvem os conteúdos estudados. Desde o ensino

\footnotetext{
' Professor do Instituto Federal de Educação. Ciências e Tecnologia do Amazonas-IFAM, ManausAm.sarley.silva@ifam.edu.br

${ }^{2}$ Professor da Universidade do Estado do Amazonas-UEA, Tefé-AM.jbcarvalho@uea.edu.br
} 
fundamental os professores geralmente trabalham com as figuras e objetos geométricos, um dos exemplos é o estudo de seus elementos e suas propriedades. As Seções Cônicas são curvas planas obtidas por intersecção de um cone circular reto de duas folhas, com um plano, dependendo do posicionamento do plano ao interceptar a superfície cônica, podemos obter três tipos de cônicas: elipse, Parábola e hipérbole. É possível trabalhar de maneira dinâmica nos permite visualizar e apresentar conceitos geométricos como medidas, propiedades, entre outros.

O estudo das figuras geométricas cónicas é de grande importância no Ensino Médio, visto que é um conteúdo que despertou interesse, e de grande importância desde tempos antigos, e o que observamos é que esse estudo muitas vezes se resume apenas a aplicação de formulas, cálculos. O seu estudo mostra o quanto o seu papel foi e é relevante para as diversas áreas do conhecimento.

Nesta abordagem, o objetivo deste estudo é desenvolver e implementar uma metodología baseada na construção de figuras geométricas envolviendo cónica visando atrair a atenção dos educandos como recurso pedagógico para a aprendizagem da Matemática, como forma de promover o raciocínio e o desenvolvimento do aluno.

As medidas e as formas geométricas estão interligadas ao desenvolvimento de conceitos. "É importante destacar que a Matemática deverá ser vista pelo aluno como um conhecimento que pode favorecer o desenvolvimiento do seu raciocinio, de sua sensibilidade expressiva, de sua sensibilidade estética e de sua imaginação" (BRASIL/PCN, 1997). Segundo o documento supracitado, os conceitos geométricos constituem parte importante do currículo de Matemática no ensino, porque, por meio deles, o aluno desenvolve um tipo especial de pensamento que lhe permite compreender, descrever e representar, de forma organizada, o mundo em que vive. $O$ trabalho com noções geométricas contribui para a aprendizagem de números e medidas, pois estimula o educando a observar, perceber semelhanças e diferenças, identificar regularidades e viceversa (BRASIL/PCN, 1997, p. 56).

O autor afirma que:

As construções geométricas possibilitam o desenvolvimento das habilidades motoras do educando, através do manuseio do material de desenho e representação dos traçados. Possibilita também o desenvolvimento do raciocinio lógico-dedutivo, da organização e da construção de estrategias pautadas nos 
conhecimentos prévios, além de propiciar a materialização de situações abstratas. (SOUZA, 2013, p. 7)

Conforme Castoldi e Polinarski:

Com a utilização de recursos didático-pedagógicos, pensa-se em preencher as lacunas que o ensino tradicional geralmente deixa, e com isso, além de expor o conteúdo de uma forma diferenciada, fazer dos alunos participantes do processo de aprendizagem". (CASTOLDI \& POLINARSKI, [8], p. o2)

Ao realizar atividades que envolvam Construções Geométricas em sala de aula o educador pode beneficiar os educandos no desenvolvimento de seu raciocínio lógico e matemático, pois esse tipo de atividade explora os conhecimentos já adquiridos e estimula a compreensão e o aprofundamento de novos conhecimentos matemáticos.

Segundo Wagner:

As construções geométricas continuam até hoje a ter grande importância na compreensão da matemática elementar. Seus problemas desafiam o raciocínio e exigem sólido conhecimento dos teoremas de geometria e das propriedades das figuras e não é exagero dizer que não há nada melhor para aprender geometria do que praticar as construções geométricas. (WAGNER, 2009,

No trabalho das construções de cónicas em grupo o professor promove interação da turma com outro grupo de colegas e contribui para o desenvolvimento da iniciativa, aumento do interesse, da curiosidade, da capacidade de análise e da reflexão dos conceitos matemáticos. São atividades assim que potencializam a motivação dos alunos para a aprendizagem de conceitos matemáticos que eles consideram complexos e difíceis.

Ao considerar a importancia das cónicas geométricas no contexto da educação em Matemática, percebemos a necessidade de trabalhar com figuras concretas na explanação dos conteudos. Nesta situação, justifica-se a escolha pelas cónicas geométricas por se tratar de um campo da Matemática mediada por definiçôes, propiedades e estruturas. Acredita-se que a construção das cônicas: elipse, parábola e hipérbole, tornam a aprendizagem significativa entre aluno e material, criando situações estimulantes, promovendo a autonomia intelectual do aluno.

\section{FUNDAMENTAÇÃO TEÓRICA}

A história das cônicas e suas curvas estão associadas ao nome de Apolônio que nasceu na cidade de Perga, século III. a.C. (EVES,1997. p.198). O qual extraiu essas 
curvas de uma superfície cônica, mediante seções planas, denominação comum de seções cônicas. Os nomes elipse, parábola e hipérbole, foram tirado da termologia Pitagórica IV sec. a.C. (EVES,1997. P.199).

Sua obra prima, seções cônicas é composta de oito volumes, da obra original sobreviveram sete volumes, sendo quatro escritos em grego e traduzidos em árabe por Tthabit Ibn Qurra, 826 a 90I no sec. IX. (BOYER, 1999.p.199). Os três primeiros volumes são baseados em trabalhos de Euclides e o oitavo volume foi infelizmente perdido.

Em i7ıo Edmund Halley traduziu os sete volumes de seções cônicas para o latim. (BOYER,1999. P.199). Embora Apolônio, tenha sido o matemático que mais estudou e desenvolveu, as cônicas e suas curvas, na antiguidade e estas, já eram conhecidas pelos percussores: Manaecmo, Aristeu e Euclides. (EVES,1997. p.198).

Manaecmo por volta de 350 a.C, atribuiu a invenção das curvas elipse, parábola e hipérbole, por ele construída mecanicamente e utilizada na resolução do clássico problema de duplicação do cubo. Apolônio mostra que, de um único cone podem ser obtidas três espécies de seção cônica, bastando para tal fazer variar a inclinação do plano. As cônicas são curvas planas obtidas por intersecção de um cone circular reto de duas folhas, com um plano. Dependendo do posicionamento do plano ao interceptar a superfície cônica, podemos obter três tipos de cônica: elipse, parábola e hipérbole. (EDWALDO, Bianchini,1998. P.578).

Os conceitos das figuras planas exposto a seguir tem a finalidade de informar de quais figuras elas surgiram e como.

\section{TIPOS DE CÔNICAS}

\section{Os Tipos de Cônicas são: Elipse, Hipérbole e Parábola.}

Parábola: Conforme Leithold (1994) Parábola é um conjunto de pontos em um plano, equidistantes de um ponto e de uma reta fixos.

Hipérbole: Chama-se hipérbole o conjunto dos pontos $\mathrm{P}$ do plano, tais que a diferencia, das suas distancias a dois pontos fixos do plano e uma constante. (Giovanni, 1993). b

Elipse: É curva que se obtém ao interceptar um cone por um plano que não é paralelo as geratrizes. (IEZZI,1993). 
Fig.r: As Cônicas em um Cone Circular de duas folhas.

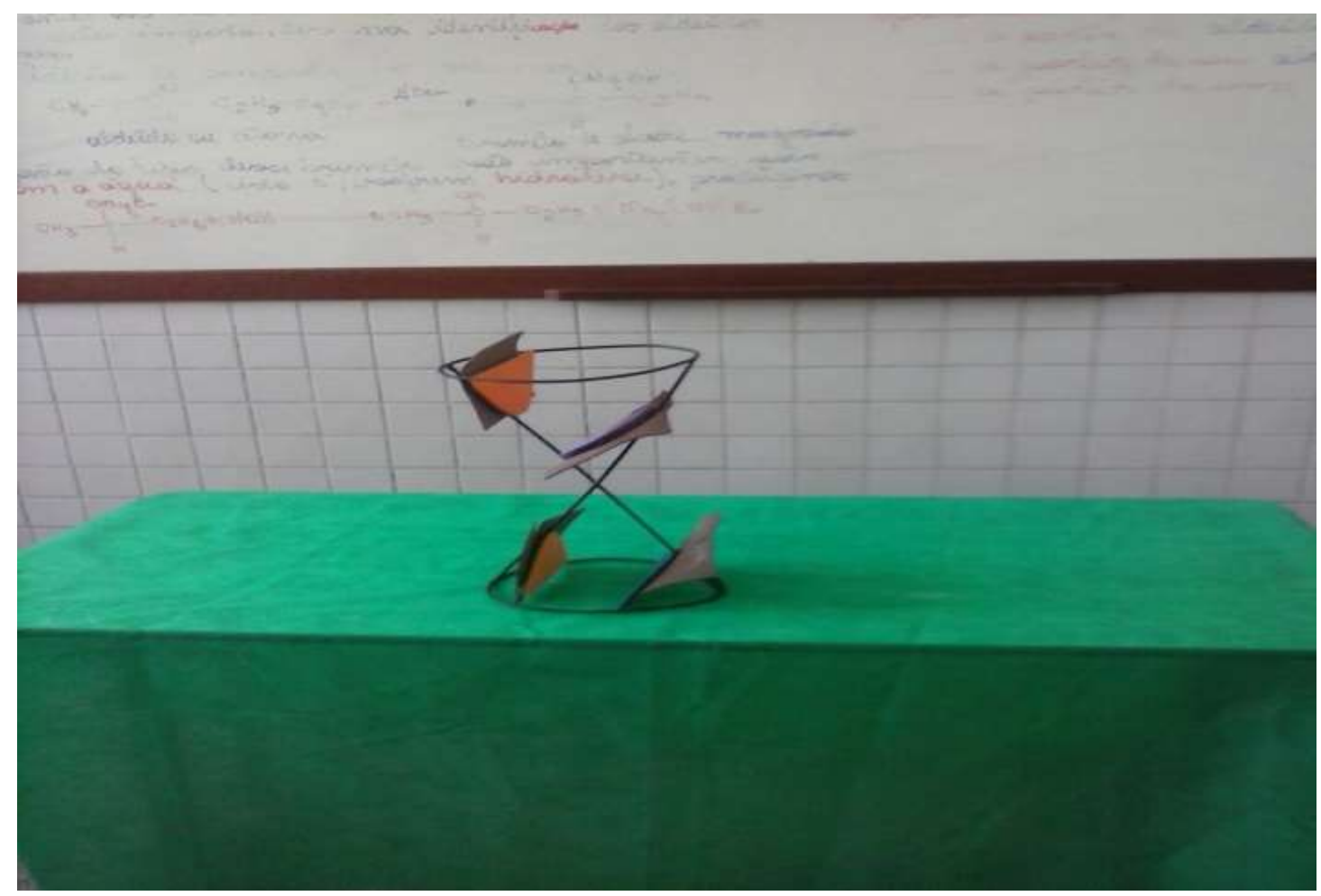

Fonte: Autoral

\section{METODOLOGIA}

O estudo das cônicas: elipse, parábola e hipérbole, foram feito através do Método Dedutivo proposto pelo Filósofo e Matemático René Descartes século XVI. Pela regra da evidência: "Pressupõe que só a razão é capaz de levar ao conhecimento verdadeiro jamais acolher alguma coisa como verdadeira que não seja evidentemente como verdadeira, isto é, de evitar cuidadosamente a precipitação e a prevenção." (ABAGNADO,2003.p.Io2).

O método dedutivo tem o objetivo de explicar o conteúdo das premissas que quando verdadeiras levam a conclusões verdadeiras, visto que pelo raciocinio, analise geral para o particular chega-se a uma conclusão, ou seja, a proposta esta dentro da pergunta.

A forma de raciocínio utilizada nesta pesquisa foi o silogismo, construção lógica que a partir de duas premissas, retira uma terceira logicamente decorrente das duas primeiras, denominada conclusão (GIL, 1999, LAKATOS, MARCONI, 1993). Se todas as premissas são verdadeiras, a conclusão é verdadeira. 
Aqui definiu-se segundo IEZZI(1993,p.I) que proposição é todo conjunto de palavras ou símbolos que exprimem um pensamento de sentido completo verdadeiro.Premissa é cada uma das preposições com informações essenciais que servem de base para á conclusão. Uma premissa é uma sentença que afirma algo (positivo ou negativamente), no contexto de um silogismo.Para representar este estudo segue abaixo,exemplo de raciocínio dedutivo utilizado para construção das cônicas.

or Etapa Afirmação Geral ELIPSE, PARÁBOLA E HIPÉRBOLE podem ser construídas (premissa maior).

02 Etapa Afirmação Particular Elipse, Parábola, Hipérbole são Cônicas. (premissa menor).

03 Etapa Logo, AS CÔNICAS podem ser construídas através da definição (conclusão).

I e 2 são premissas que levam á conclusão 3 .

I,2 e 3 formam o silogismo

A construção das figuras cônicas ocorreu através da curvatura em ferro 3/16 e medidas que seguiam regra da definição de cônicas dos pontos equidistantes, essa atividade e voltada para os alunos do $3^{\underline{o}}$ ano do ensino médio da Escola Estadual Deputado Armando de Souza Mendes em Tefé.

$\mathrm{Na}$ construção das cônicas foram utilizados os seguintes matérias: ferro $3 / \mathrm{l} 6$, solda, tinta amarela, tinta vermelha, emborrachado, nylon, barbante, trena de medição. $\mathrm{O}$ estudo desenvolveu-se através da construção das cônicas pelo método de curvatura plana, tendo como apoio os seguintes materiais: construção de cônica com régua e compasso e método de dobradura. (GIOVANNI,2005. P.106,122 e 128).

Foi construida com o objetivo de estudar definição, diferenciação e identificação de seus elementos. Essas atividades foram direcionadas para os alunos do $3^{\circ}$ ano do ensino médio da escola $\mathrm{GM}_{3}$ em Tefé, envolveu-se no trabalho fotografo e educandos na construção das cônicas: elipse, parábola e hipérbole.

\section{CONSTRUÇÃO GEOMÉTRICA DA PARÁBOLA PELO MÉTODO DE CURVATURA PLANA.}

DEFINIÇÃO: Considere em um plano uma reta $\mathrm{d}$ e um ponto $\mathrm{F}$ não pertencente á reta d. Parábola conjuntos de todos os pontos a um plano equidistante de $\mathrm{F}$ tais que a distância de PF=PP`. (IEZZI,1993. P.I78). 


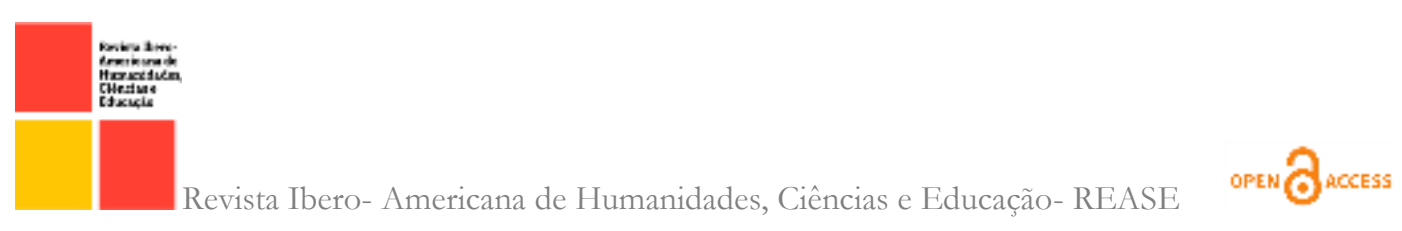

Fig. 2: Parábola

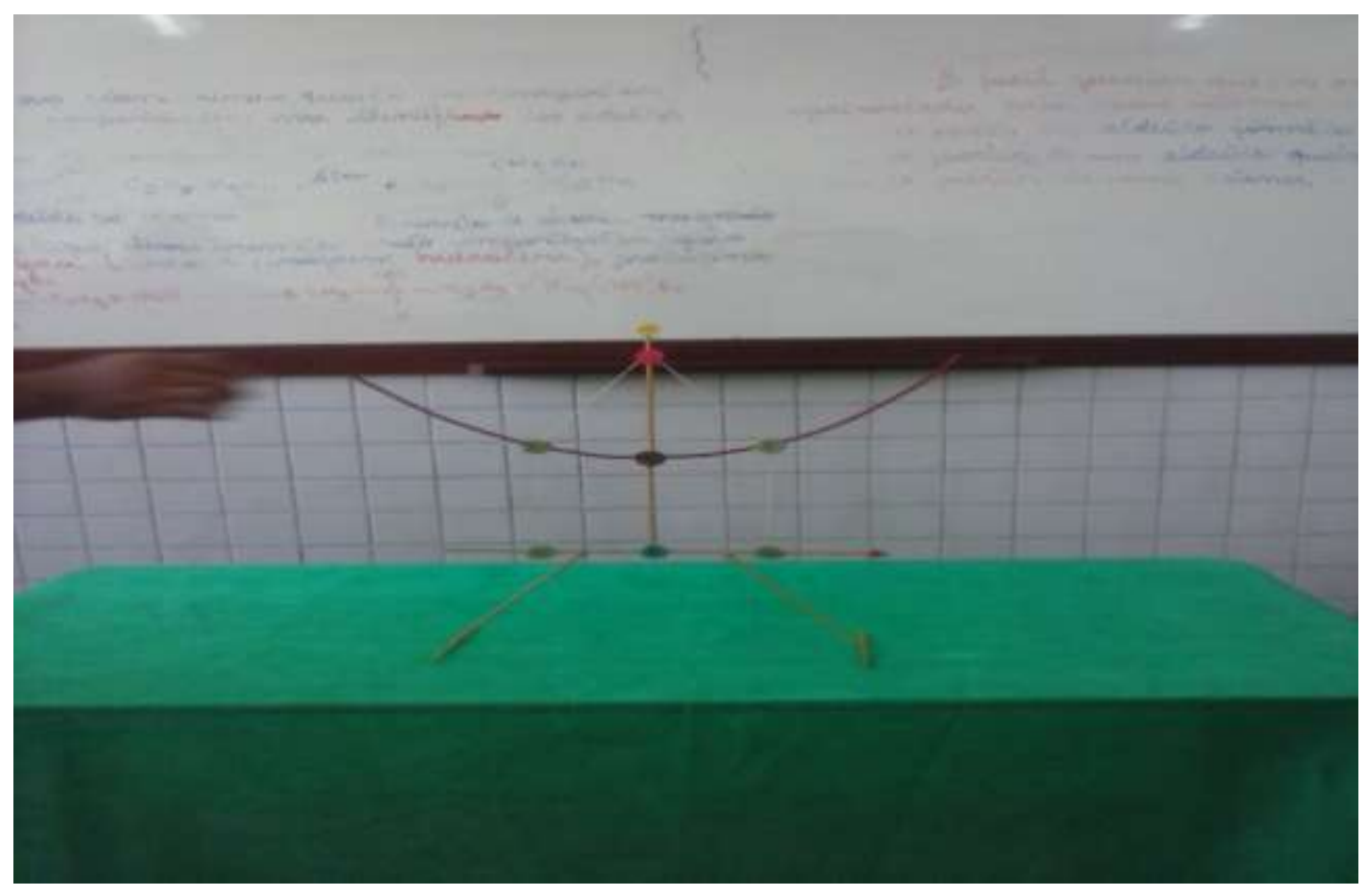

Fonte: Autoral

Referente a Parábola, há várias propostas, entretanto, estudou-se apenas uma: A 1611 construção de uma parábola. Seguindo a equação de definição da parábola PF=PP`.

Dada uma reta $\mathrm{d}$ em um plano e um ponto $\mathrm{F}$ não pertencente a reta $\mathrm{d}$, marca-se um ponto $\mathrm{A}$ no meio da reta diretriz e um ponto $\mathrm{V}$ no meio das distancias dos pontos $\mathrm{FA}$, fazendo uma curvatura em um ferro de $56 \mathrm{~cm}$, as distancias dos pontos de V A e V F tendo medidas iguais, marca o ponto $\mathrm{P}$ do lado direito da parábola tomando ponto seguindo a definição $\mathrm{PF}=\mathrm{PP}$ '.

Em seguida continuou-se curvando e ligando novos pontos com medidas que se distanciam iguais pela definição, FP $=\mathrm{PP}$. Continuando o processo de construir infinitos pontos, seguindo essas condições de FP = PP `, vai gera uma curva aberta de um ramo chamada parábola.

As medidas utilizadas na construção da parábola foram as seguintes:FVA medida de $24 \mathrm{~cm}, \mathrm{FV}$ medida de $12 \mathrm{~cm}$,VA medida de $12 \mathrm{~cm}$ e a curva com $56 \mathrm{~cm}$. Tendo como objetivo de facilitar o aprendizado,trabalhando a percepção dos alunos na identificação dos seus elementos: $\mathrm{F}$ - foco, $\mathrm{d}$ - diretriz, $\mathrm{P}$ - parâmetro é a distancia de entre $\mathrm{F}$ á $\mathrm{d}, \mathrm{V}$ vértice e Relação notável: $\mathrm{VF}=\mathrm{P} \div 2$. 


\section{CONSTRUÇÃO DA HIPÉRBOLE PELO MÉTODO DE CURVATURA PLANA. DEFINIÇÃO:}

Considera num plano dois pontos distintos que chamamos de $F_{\mathbf{r}}$ e $F_{2}$ e $2 \mathbf{a}$ um número real maior que a distancia de Fr á F2. Chamamos de hipérbole o conjunto dos pontos do plano tais que a diferença das distancias dos pontos PFI-PF2= 2a. (IEZZI,1993. P.174).

Fig. 3: Hipérbole.

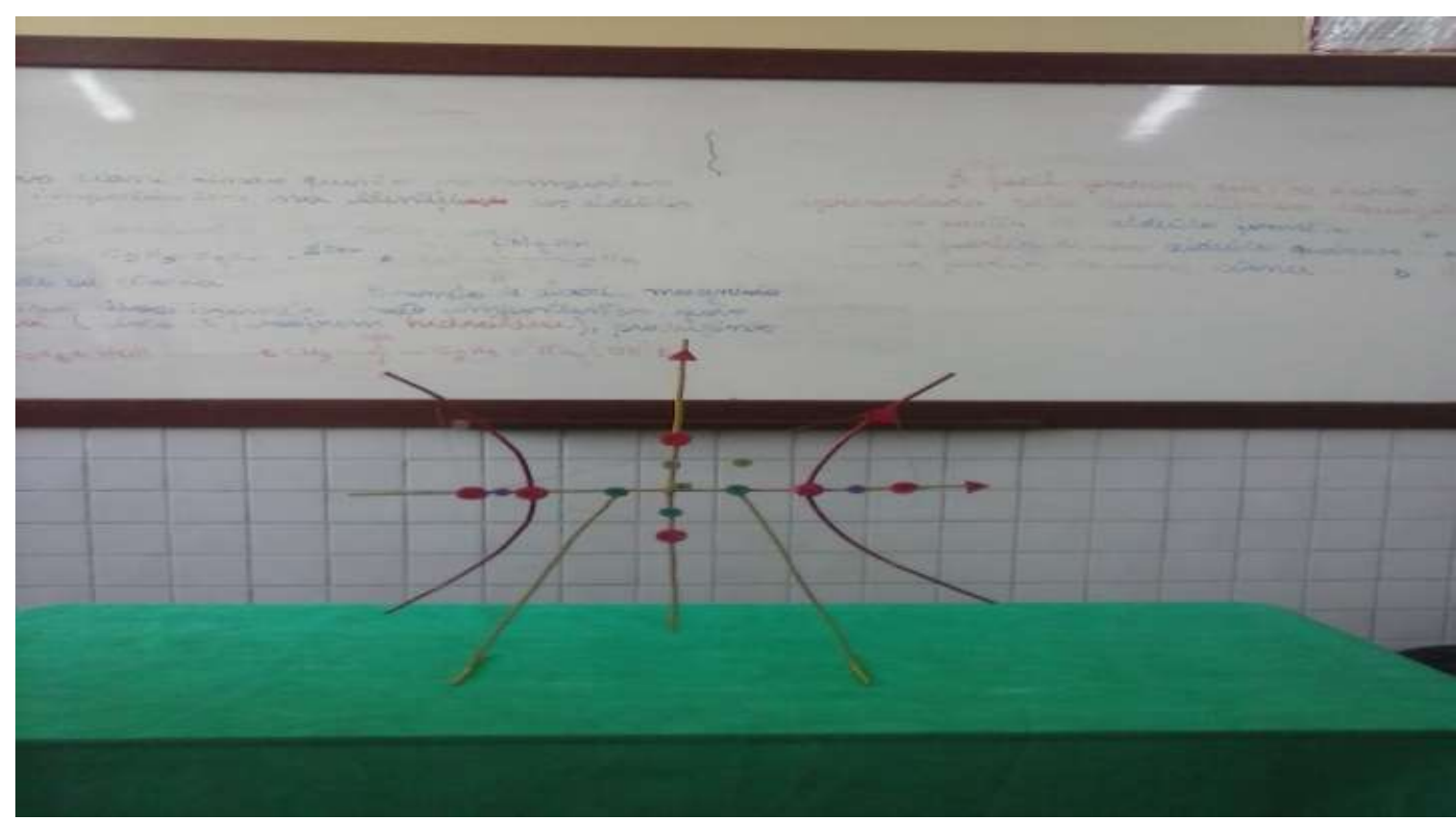

Fonte: Autoral

Dada uma reta d, marca-se dois pontos $F_{1}$ e F2, antes de Fi marque o ponto Ar e antes de $\mathrm{F}_{2}$ o ponto A2, fazendo uma curvatura em dois ramos em cima dos pontos Ar e A2, marcar um ponto $P$ no ramo direito da hipérbole tomando ponto seguindo a definição $\mathrm{PF}_{1}-\mathrm{PF}_{2}=\mathbf{2 a}$.

Em seguida continuou-se curvando e ligando novos pontos com medidas que se distanciam iguais pela definição $\mathbf{P F}_{\mathbf{I}}-\mathrm{PF}_{\mathbf{2}}=$ 2a. Continuando o processo de construir infinitos pontos, seguindo essas condições de $\mathrm{PF}_{\mathrm{I}}-\mathrm{PF}_{2}=2 \mathrm{a}$, vai gera uma curva aberta de dois ramos chamada hipérbole.

As medidas utilizadas na construção da hipérbole foram as seguintes: $\mathrm{B}_{1} \mathrm{~B}_{2}$ medida de $20 \mathrm{~cm}$, AIA2 medida de $20 \mathrm{~cm}$ e ramos com medida de $50 \mathrm{~cm}$. Tendo como objetivo de facilitar o aprendizado, trabalhando a percepção dos alunos na identificação dos seus elementos: $F_{1}$ e $F_{2}$ - focos, $\mathrm{O}$ - centro, $\mathrm{A}_{1} \mathrm{~A}_{2}$ - eixo real ou transverso, $\mathrm{B}_{1} \mathrm{~B}_{2}$ - eixo 
imaginário, 2 c - distancia focal, 2 a - medida do eixo real, 2 b - medida do eixo imaginário, $\mathrm{c} \div \mathrm{a}$ - excentricidade.

\section{CONSTRUÇÃO GEOMÉTRICA DA ELIPSE PELO MÉTODO DE CURVATURA} PLANA

DEFINIÇÃO: Considere em um plano dois pontos distintos que chamamos $F_{\mathbf{r}}$ e $F_{2}$ e $\mathbf{2}$ a um numero real maior que a distancia de Fr e F2. Chamamos de elipse o conjunto dos pontos $\mathrm{p}$ do plano tais que a soma das distancias de $\mathrm{PF}_{\mathbf{I}}+\mathrm{PF}_{\mathbf{2}}=\mathbf{2}$ a. (IEZZI,1993.168).

Fig. 4: Elipse.

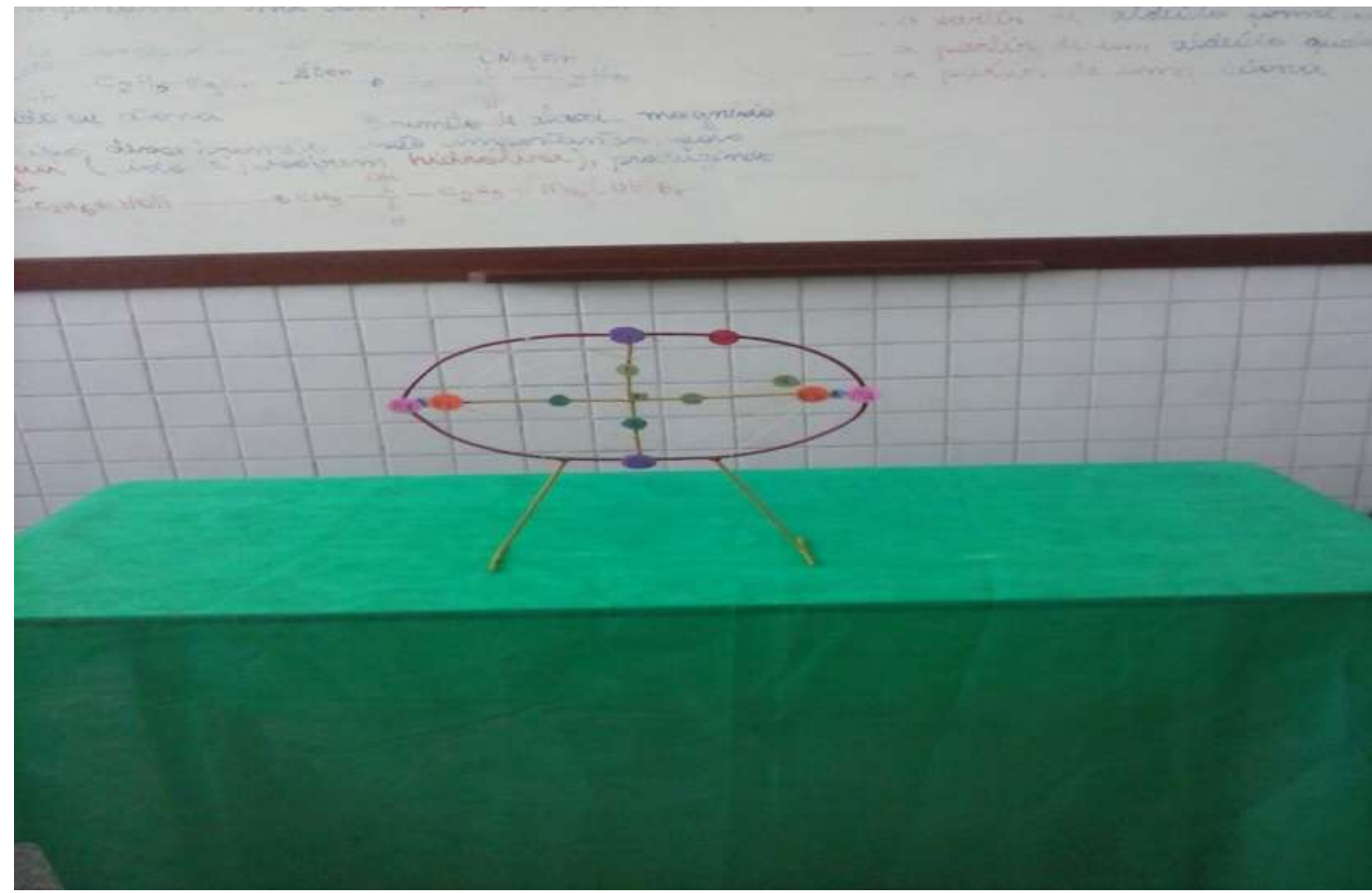

Fonte: Autoral

Dada uma reta d, marca-se dois pontos $F_{1}$ e $F_{2}$, após $F_{1}$ marque o ponto Ar e após $\mathrm{F}_{2}$ o ponto A2, fazer curvatura em $\mathrm{A}_{1}$ e $\mathrm{A}_{2}$, marcar um ponto $\mathrm{P}$ do lado direito da curva da elipse tomando novos pontos seguido pela definição a distancia $P_{\mathbf{I}}+\mathrm{PF}_{\mathbf{2}}=\mathbf{2 a}$. Em seguida continua curvando e ligando novos pontos com medidas que se distanciam iguais pela definição $P_{\mathbf{I}}+\mathrm{PF}_{2}=$ 2a. Continuando o processo de construir infinitos pontos, seguindo essas condições de $\mathrm{PF}_{1}+\mathrm{PF}_{2}=2 \mathrm{a}$, vai gerar uma curva fechada chamada elipse.

As medidas utilizadas na construção da elipse foram as seguintes: AıA2 medida 35 $\mathrm{cm}, \mathrm{B}_{1} \mathrm{~B}_{2}$ medida $20 \mathrm{~cm}$ e curva de $84 \mathrm{~cm}$. Tendo como objetivo de facilitar o aprendizado,trabalhando a percepção dos alunos na identificação dos seus elementos: Fi e 
$\mathrm{F}_{2}$ - focos, O - centro, $\mathrm{A}_{1} \mathrm{~A}_{2}$ - eixo maior, $\mathrm{B}_{1} \mathrm{~B}_{2}$ - eixo menor, $2 \mathrm{c}$ - distancia focal, 2 a medida do eixo maior, $2 \mathrm{~b}$ - medida do eixo menor, $\mathrm{c} \div \mathrm{a}$ - excentricidade e relação notável $a^{2}+b^{2}=c^{2}$.

\section{RESULTADOS E DISCUSSÕES}

Através da construção de figuras de forma concreta, foi trabalhada a maneira de perceber acompanhada de recursos cognitivos (conceito, observação, diferenciação, identificação e aprendizagem em grupo).

Participaram do estudo alunos do $3^{\circ}$ ano do ensino médio da Escola Estadual Deputado Armando de Souza Mendes em Tefé-AM. A atividade foi desenvolvida em duas etapas a primeira etapa execução e construção das figuras, exploração do conteúdo e da métodologia a ser utilizada.

A segunda etapa a turma foi distribuida em seis grupos, cada um contendo cinco alunos de posse dos seguintes matérias de manipulação: ferro 3/16,cola, barbante, emborrachado, nylon, régua e trena de medição da construção das cônicas, foi possivel os alunos diferenciarem e identificarem cada uma aplicando seus conhecimentos,e trabalhando a definição para construção das figuras geométricas, verificou-se que pela definição fazendo curvatura em ferro $3 / 16$ e estabelecendo medidas nos pontos equidistantes que seguem a definição torna-se simples a construção, houve a inter-relação professor-aluno e um interesse maior com relação a disciplina. Antes da aplicação do projeto foram feitas as seguintes perguntas para os alunos: O que são cônicas? Conceito de parábola e a história das cônicas e suas curvas estão associado a qual nome?Quem mais desenvolveu o estudo? Como verificação do conhecimento dos alunos em relação ao conteúdo já ministrado pelo professor responsável pela turma, verificou-se que dos 35 alunos, somente is responderam alguma informação sendo que com imprecisão,depois do trabalho executado todos responderam,passaram a ter melhor compreensão trabalhando com construção de cônicas. Tiveram melhor esclarecimento em relação aos seus elementos, verificaram a importância do conceito em relação a construção de figuras planas.Segundo o professor responsável a metodologia utilizada contribuiu muito com a turma, passaram a ter melhor desempenho.Foi confirmado que é possível construir 
cônicas, a partir da definição e materiais concretos mediada pela aprendizagem significativa.

Fig. 5: Construção da Hipérbole.

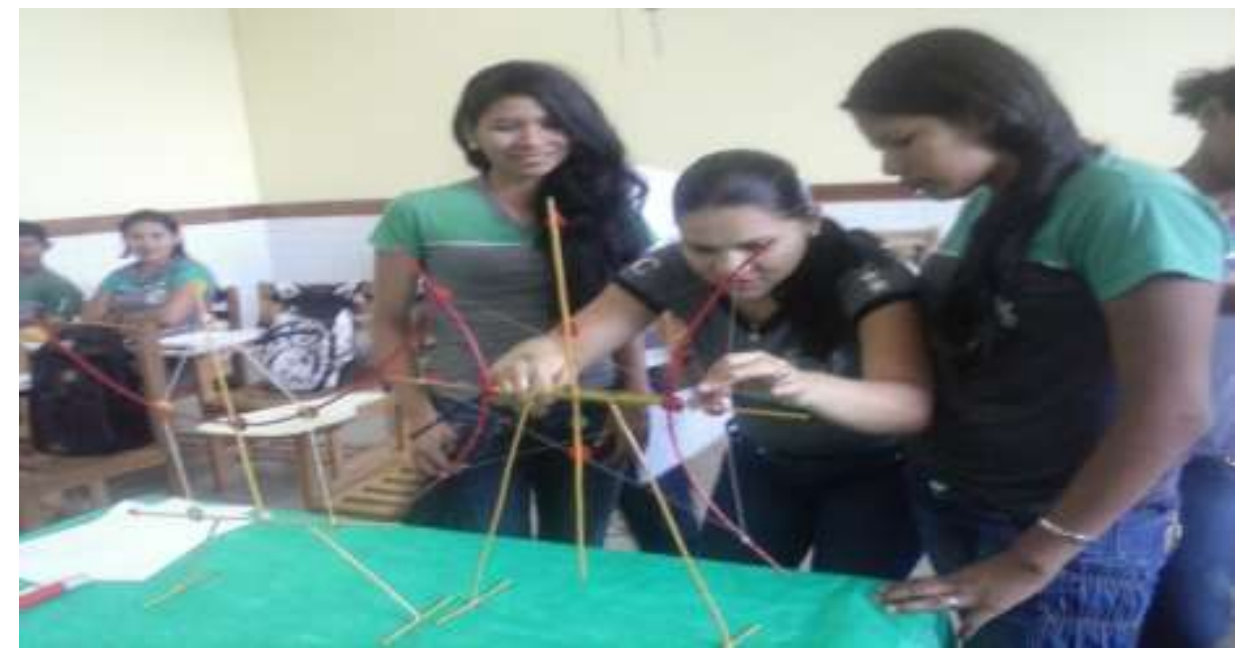

Fonte: Autoral

A figura. 05 mostra os grupos de alunos do $3^{\circ}$ ano do Ensino Médio da Escola Estadual Deputado Armando de Souza Mendes em Tefé, construindo a hipérbole, fazendo medição dos pontos que se distanciam igualmente segundo a equação da definição da hipérbole. Verificaram que através do contato com material concreto seguindo corretamente a definição de cônicas facilita o aprendizado.

Fig. 6: Construção da Parábola

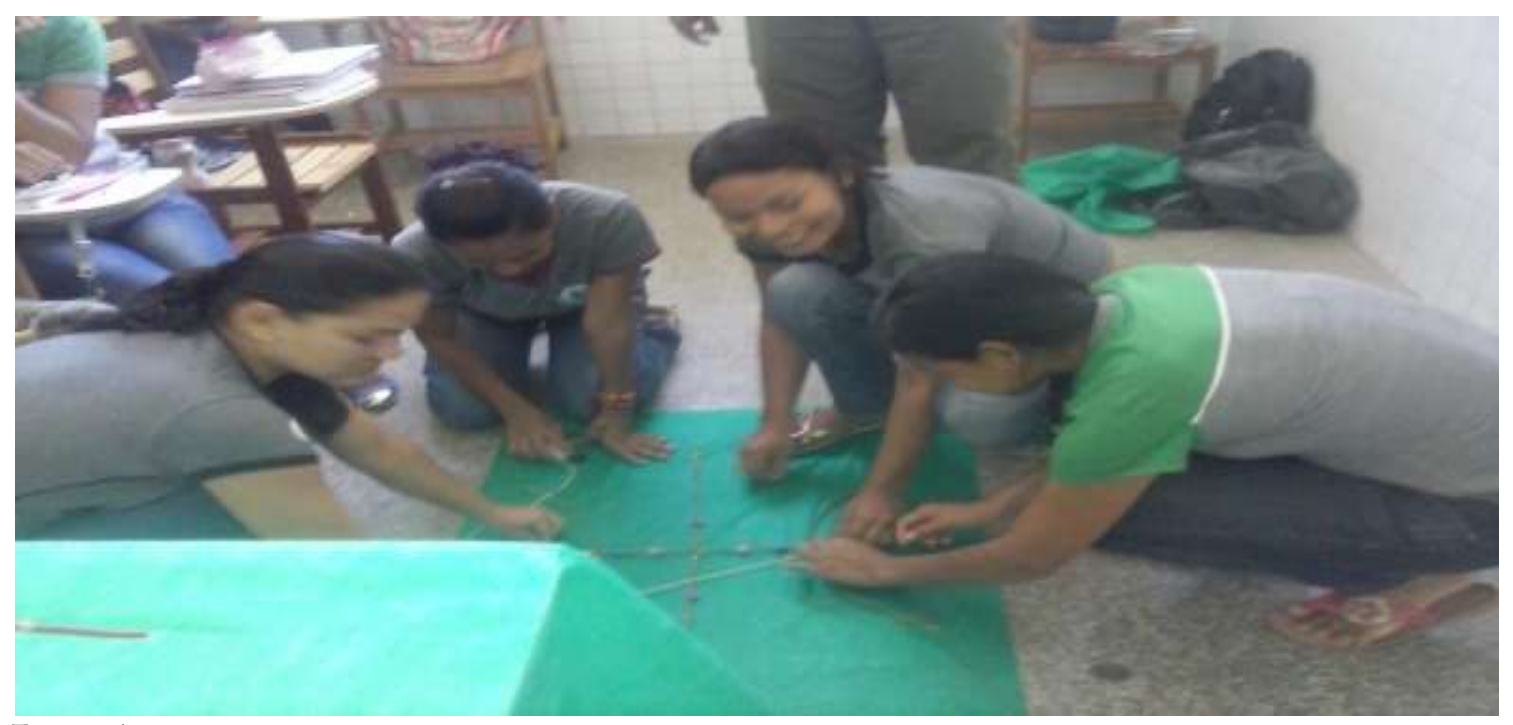

Fonte: Autora 
Na fig.o6 Os educandos trabalham a construção da parábola conforme medidas já citadas, desenvolvendo o espírito de trabalho em equipe e aprendendo a confeccionar material didático concreto.

Fig. 7: Construção da Elipse.

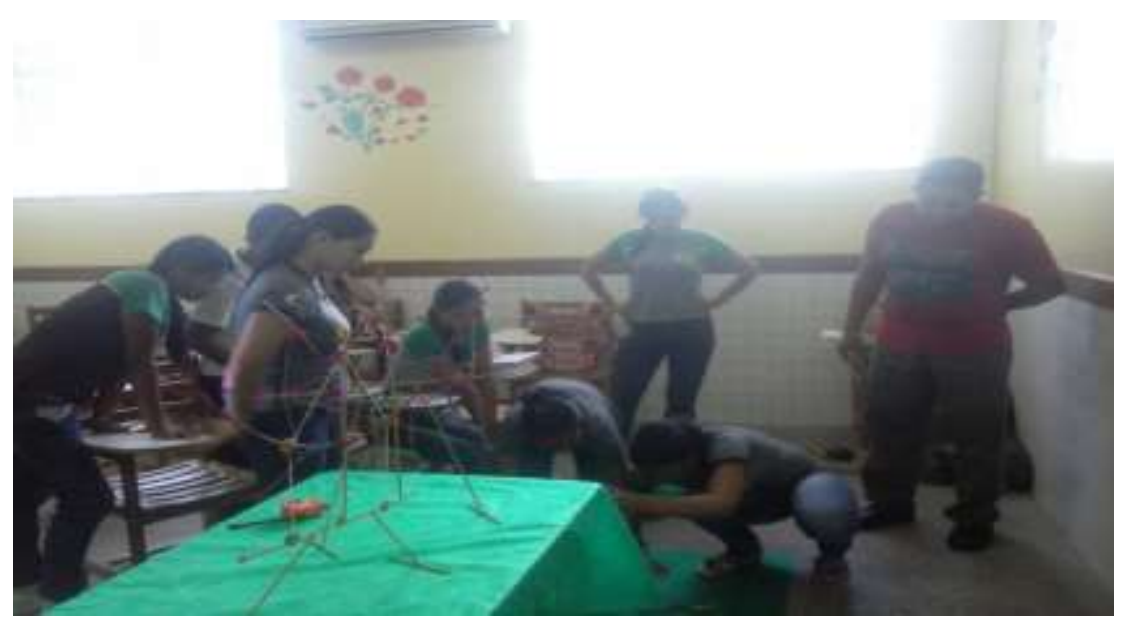

Fonte: Autoral

$\mathrm{Na}$ fig.o7 Os alunos trabalham conceitos de cônicas na construção da elipse e verificando a localização dos seus elementos. Constataram que realmente é possível construir figuras planas, pelo método dedutivo a partir da definição, atuaram de forma ativa e coletiva na construção do conhecimento. As construções geométricas auxiliam na compreensão de conceitos geométricos, bem como desenvolvem habilidades para executar procedimentos,estimulam a criatividade do aluno, quando é dada a ele a oportunidade de imaginar e criar as construções de figuras planas.

Fig. 8: As cônicas.

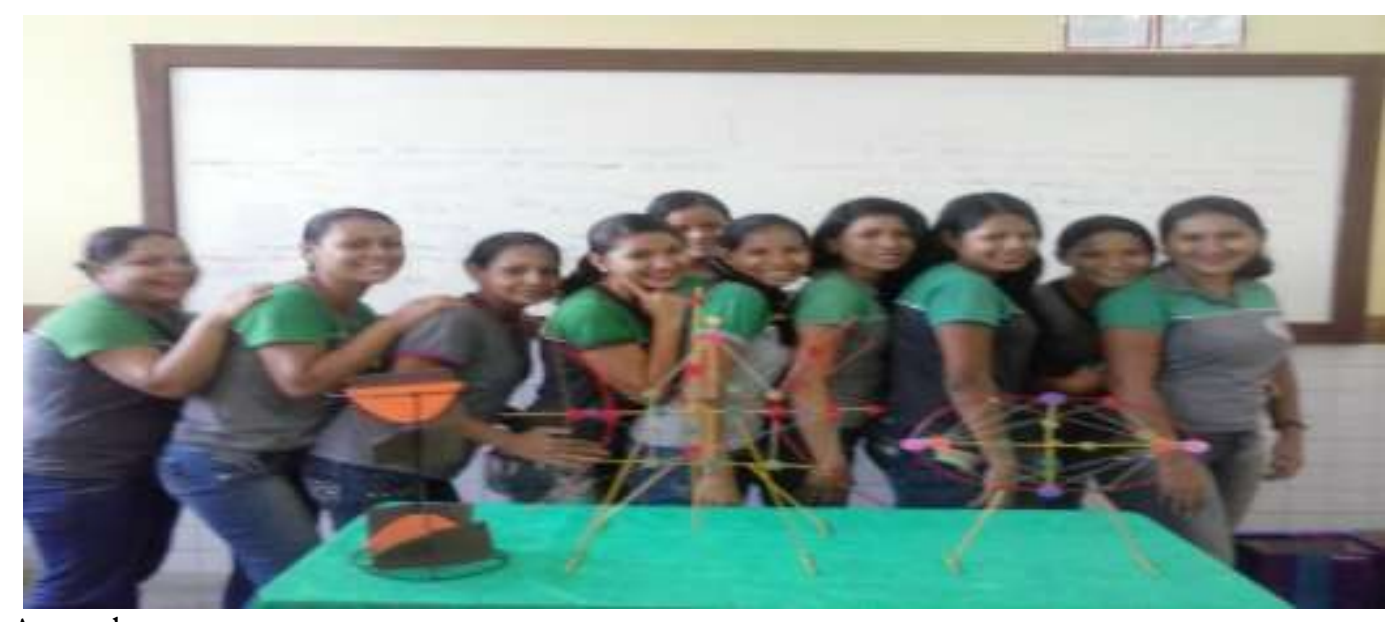

Fonte: Autoral 
Enquanto na fig.08 mostrar a turma de alunos $3^{\text {o }}$ ano do Ensino Médio da Escola Estadual Deputado Armando de Souza Mendes em Tefé, diante das figuras cônicas: Elipse, Hipérbole e Parábola.

\section{CONSIDERAÇÕES}

Por meio da construção das cônicas, a partir da definição foi possível estudar seus elementos e propriedades. Durante o desenvolvimento do trabalho no estudo das cônicas os educandos trabalharam definição, construção, diferenciação e identificação, observou na prática a construção das figuras pelo método dedutivo a partir da definição curvatura em figuras planas.

O trabalho foi de suma importância, pois, os alunos aprenderam a utilizar os conceitos matemáticos e construir figuras, trabalharam em equipe e participaram na prática. Sendo assim o educador matemático não deve medir esforços em aceitar o desafio de procurar trabalhar com construções de cônicas e desenvolver atividades que possam despertar o interesse dos alunos e aumentar a participação durante as aulas de matemática.

Os objetivos expostos nessa pesquisa foram alcançados com êxito ao possibilitar construir figuras geométricas pela definição e fazer uso das figuras geométricas como recurso pedagógico para a aprendizagem da Matemática, como forma de promover o raciocínio e o desenvolvimento do aluno. Esse estudo expôs que é possível trabalhar medição, identificação de elementos e diferenciação de figuras geométricas através da observação direta.

Dessa forma, a utilização de materiais concretos no ensino-aprendizagem das cónicas auxilia os educandos através da visualização dos conceitos geométricos a aprender, a compreender, a entender e a fixar conceitos importantes de geometría analítica. Essa abordagem favoreceu, a motivação e o interesse dos educandos no processo de ensino aprendizagem em cónicas.

Consideramos que o educador deve procurar alternativas que motivem os seus alunos, desenvolvendo neles o senso de confiança, organização, concentração. Por tanto, sair do comportamento tradicional e adentra ao inovador, no qual estimula o aluno a interagir com o objeto estudado e com as pessoas ao seu redor e assim tornar a aprendizagem interessante. 


\section{SUGESTÕES}

Como trabalhos futuros, sugere-se realizar o estudo da geometria espacial. Aplicando construções de figuras concretas juntamente com uma metodologia adequada. Enfim, incluir nos estudos figuras espaciais. Essas figuras matemáticas estão direcionadas para trabalhos futuros onde, podem ser feitas novas pesquisas sobre estudo da geometria espacial como recurso pedagógico.

\section{AGRADECIMENTOS}

Agradeço a Deus pela vida e pela força de acordar todos os dias e luta pelos meus objetivos, aos professores pela oportunidade, ensinamentos e exemplos de dedicação. A Deus, acima de todas as coisas presente. Ao meu orientador pelo acompanhamento durante as pesquisas das cônicas e suas construções geométricas, pelo apoio na elaboração deste artigo. Aos professores e colegas da Escola Estadual GM3.

\section{REFERÊNCIAS BIBLIOGRÁFICAS}

ABAGNADO, Nicola. Dicionário de Filosofia.4를 ed. São Paulo:Martins Fontes,2003.

BOYER, Carl B. História da Matemática. $2^{-}$ed.vol.ı.São Paulo:Editora Edgard Blucher Ltda,1999.

Brasil. Parâmetros Curriculares Nacionais: Matemática. Secretaria da Educação Fundamental. Brasília: MEC/SEF, 1997.

CASTOLDI, R.; POLINARSKI, C. A. A Utilização de Recursos Didático-Pedagógicos na Motivação da Aprendizagem. I Simpósio Nacional de Ensino de Ciência e Tecnologia - Programa de Pós-Graduação em Ensino de Ciência e Tecnologia, Universidade Tecnológica Federal do Paraná, 2009.

EVES, Howard Whitley. Introdução á História da Matemática. 2º ed.Campinas

São Paulo:Editora da Unicamp,1997.

EDWALDO, Bianchini. Matemática. Vol.ı.2ํe․ São Paulo:Moderna, 1998.

GIOVANNI e Jose Rui. Geometria analítica. 2º ed.São Paulo:FTD,2005.

GIL, Lakatos e Marconi. Metodologia Cientifica.3ed.São Paulo.vol.ı.São Paulo:Atlas,200o. 
Giovanni, José Ruy Aprendendo matemática: novo / José Ruy Giovanni, Eduardo Parente. - Säo Paulo: FTD, 1993.

HELENA, Maria. Matemática. Io ed.São Paulo.Atlas:2004.

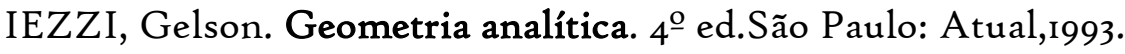

LORENZATO, Sergio. Para Aprender Matemática. $3^{\circ}$ ed.Rev. Campinas São Paulo: Autores Associados.2010.

Leithold, Louis. O Cálculo com Geometría Analítica. Vol. Iae edição, editora HARBRA, São Paulo,1994.

SOUZA, R. D. O Resgate do Ensino das Construções Geométricas na Educação Básica. Illhéus: Dissertação de Mestrado Universidade Estadual de Santa Cruz, 2013.

WAGNER, E. Construções Geométricas. Rio de Janeiro: SBM, 2007. 